\title{
Significato e origine di perplimere: nota di aggiornamento
}

\author{
Luisa di Valvasone
}

PUBBLICATO: 24 FEBBRAIO 2021

\section{Quesito:}

Rispondiamo alle domande intorno al verbo perplimere a alla sua appartenenza alla lingua italiana con un aggiornamento della risposta di Marco Biffi da tempo pubblicata sul nostro sito.

\section{Significato e origine di perplimere: nota di aggiornamento}

"Il verbo perplimere significa "essere perplesso" o "rendere perplesso", ed è entrato nella nostra lingua in un passato recente, ma con un percorso particolare che ne ha limitato l'ambito d'uso e che ne ha pertanto impedito, almeno per ora, l'ingresso nei vocabolari di lingua italiana (nei quali non è attualmente registrato).

L'impiego del verbo perplimere è dovuto alla prosa creativa del comico Corrado Guzzanti, che lo ha lanciato nei primissimi anni Novanta, nella trasmissione televisiva "Avanzi". La parola venne inserita in uno dei dialoghi fra il personaggio Rokko Smitherson e Serena Dandini, ed ebbe talmente successo che fu più volte riutilizzata nella trasmissione, con ricchi esempi nella coniugazione (perplimere, perplimo, perplimete, perplei, perplime[re]) e nelle varianti (perplerre).

Molte furono le parole inventate da Rokko Smitherson (regista romano di "filmaggi de paura"), un personaggio che basava la sua comicità satirica proprio sui giochi di parole e su neoconiazioni allusive (sospensionismo, su astensionismo; antiproibizionale, su antiproibizionista; sopravvolare, su sorvolare; cartone animale, su cartone animato; psicoanale, su psicoanalista; ecc.). Fra le molte innovazioni linguistiche perplimere attecchì più facilmente nella lingua comune a causa della sua perfetta adeguatezza morfologica, che tra l'altro colma anche una lacuna lessicale della nostra lingua: il verbo è infatti spontaneamente riconducibile dai parlanti italiani al participio passato perplesso (sulla base di verbi come comprimere/ compresso; sopprimere / soppresso, ecc.); e del resto manca in italiano un verbo che renda in modo sintetico l'azione dell'essere o del rendere perplessi, per cui il neologismo si incunea perfettamente nel nostro sistema linguistico.

Probabilmente per questa sua funzionalità nel coprire un vuoto morfologico e semantico (che l'italiano eredita dal latino), sulla scia della trasmissione la parola ebbe una notevole e crescente fortuna, seppure in contesti informali e per lo più in accezione ironica; e, del resto, nonostante l'origine peculiare, perplimere ha resistito a lungo nella nostra lingua, tanto che recentemente se ne è persa anche la sfumatura ironica, come emerge dai quesiti e dalle segnalazioni di neologismo giunti alla nostra redazione.

Effettuando una ricerca su Internet con i consueti motori di ricerca, si rileva che la parola è comparsa in alcuni dizionari amatoriali, ed è spesso presente nei messaggi di vari gruppi di discussione, tra l'altro con forti oscillazioni tra chi sostiene che il verbo non esiste e chi invece ne dà per scontata l'assimilazione nel repertorio dellitaliano e lo usa per spiegare altri fenomeni linguistici. A questo proposito, e per rispondere a chi ci domanda come si possa fare entrare il verbo perplimere nei vocabolari, va ricordato che la grammatica e la lessicografia registrano, raccolgono, spiegano e inseriscono in un sistema organico fenomeni che hanno raggiunto una certa rilevanza nella lingua: perplimere nasce certamente come parola effimera, ma la sua tenace persistenza la rende linguisticamente interessante; per cui non se ne può negare, come fanno alcuni, l'esistenza, ma se sia o meno destinata ad entrare nei vocabolari è una risposta che può essere data soltanto dalla continuità e dall'ampiezza della sua diffusione nei prossimi anni.

Marco Biffi (6 giugno 2003)" 
$\mathrm{V}$ erifichiamo a distanza di anni la tendenza d'uso e la situazione lessicografica di perplimere. Come scrive Paolo D'Achille in un articolo del 2005, il verbo potrebbe essere inserito nella lista di quelle retroformazioni italiane che derivano da aggettivi o nomi interpretati dai parlanti come participi (nel nostro caso l'aggettivo perplesso), come nei casi di candidare derivato da candidato e deodorare tratto da deodorante (Paolo D'Achille, Le retroformazioni in italiano, in Lessico e formazione delle parole. Studi offerti a Maurizio Dardano per il suo $70^{-}$compleanno, a cura di Claudio Giovanardi, Firenze, Cesati, 2005).

Se in questi anni perplimere non è stato dal GRADIT e dal Devoto-Oli, il Garzanti invece, sulla base della scheda di Biffi, lo citava rapidamente già nel 2007, in una nota sotto la voce perplesso, per poi metterlo definitivamente a lemma nell'edizione del 2017 (marcato come "scherzoso"); un'ampia trattazione del verbo è inoltre presente nello Zingarelli a partire dall'edizione 20r3, di cui riportiamo l'intera voce, con la nota etimologica, rimasta nelle successive edizioni del dizionario:

perplimere [retroformazione creata dal comico C. Guzzanti nella trasmissione televisiva Avanzi dall'agg. perplesso interpretato come se fosse il part. pass. di un v. coniugato sul modello di opprimere 1998] A v. tr. (pass. rem. io perplèssi o perpliméi, tu perplimésti; part. pass. perplésso) - (colloq., scherz.) rendere perplesso B v. intr. e perplimersi v. intr. pron. - (colloq., scherz.) essere, diventare perplesso.

Il verbo 'perplimere' è un esempio di retroformazione, cioè di processo mediante il quale una parola deriva da un'altra che sembrerebbe un suo derivato. Infatti l'aggettivo 'perplesso' deriva dal latino perplexus, part. pass. del verbo perplectere. Invece 'perplimere' è una parola di formazione recente; è un verbo deaggettivale, cioè deriva dall'agg. perplesso (derivazione supposta per analogia con oppresso, part. pass. di opprimere, o con depresso, part. pass. di deprimere). È di uso soltanto colloquiale e generalmente scherzoso.

Recentemente, invece, perplimere è stato inserito nel repertorio dei Neologismi 2021 del Vocabolario Treccani online.

Sappiamo ormai che la fortuna del verbo si deve a Guzzanti e al programma televisivo Avanzi andato in onda dal I99r al I993; per quanto riguarda però la data di prima attestazione lo Zingarelli indica il I998 e possiamo trovare su Google libri un'attestazione nel saggio Graf militante di Marziano Guglielminetti che introduce una raccolta di saggi di Arturo Graf (1848-19I3) curata da Clara Allasia e pubblicata nel i998:

Persino la sua sostanziale estraneità alla poesia greca, da Thovez sommamente e modernamente pregiata, non gli suscita riserve; meno che mai, ed è banale dirlo, lo perplime la decisa ripresa di Leopardi, [...]. (Arturo Graf, Arturo Graf militante: saggi scelti, a cura di Clara Allasia, Torino, Scriptorium, 1998 , p.36)

Molto antecedente è un'attestazione di perplimere, emersa dalle ricerche su Google libri, in un trattato di medicina del I840, tradotto dal francese, il cui significato non è tuttavia del tutto chiaro (potrebbe significare 'reprimere' o forse 'confondere') e che potrebbe dipendere dalla lingua d'origine da cui è tradotto:

Infine il prof. Remer ammette benanche una apoplessia nervosa che paralizza istantaneamente l'azione del cervello, come quella del cuore e dei polmoni, sviluppandosi sotto l'influenza della causa morale che perplime lo spirito de' sospesi per la gola;[...]. (A. Devergie, Trattato di medicina legale teoretica e pratica, vol. III,"volto in italiano, commentato ed adattato al codice delle Due Sicilie dal Dot. Cesare Miglietta", Napoli, Puzziello, i840) 
Si tratta in ogni caso di unoccorrenza isolata, giacché tutte le altre occorrenze trovate in rete risalgono all'ultimo ventennio. La presenza crescente di attestazioni, rilevate a febbraio 202I, dimostra una discreta diffusione del verbo. Circa una ventina di occorrenze (sommando i risultati tra la forma all'infino e le forme flesse) si hanno nell'archivio della "Repubblica", anche se una parte delle attestazioni si trova in articoli che trattano appunto di neologismi della nostra lingua. Qualche centinaio le attestazioni su Google libri delle diverse forme flesse. Tra le pagine in italiano di Google troviamo 3.370 risultati per perplimere, 2I.300 per perplime (comuni espressioni del tipo "questo fatto/questa cosa mi perplime"), 4.280 per perplimo, 576 per perplimi, 372 per perplimersi, e in generale si trovano occorrenze delle forme flesse in tutti i modi e i tempi verbali dell'italiano, segno di vitalità del termine:

ho provato a scrivere una poesia che ti / spaventasse, una che ti commuovesse, una / che ti rallegrasse, una che ti immalinconisse, una / che ti ringalluzzisse, una che ti allarmasse, una che ti / sconquassasse, una che ti ammansisse una che ti perplimesse / una che ti innamorasse / e non / ce l'ho / fatta (Guido Catalano, Non ebbi la prontezza, in Ti amo ma posso spiegarti, Milano, BUR, 20I8; ma la poesia appariva già nel blog dell'autore il 7/7/2010)

Il film, pur perplimendo gli estimatori del cinema muto, è risultato comunque interessante per l'utilizzo della tecnologia digitale usata per estrapolare gli sfondi pittorici, che lo resero celebre, e riutilizzarli nella versione aggiornata, ricreando un'affine estetica. (Trovato il protagonista del remake di Nosferatu ad opera di David Lee Fisher, www.ilcineocchio.it, I4/4/2016)

Uno scambio di sguardi muti e stupiti. La cosa mi perplesse, ma anche divertì. Tanto, non c'era niente di male a dirlo. (Fabio Novel, Scatole siamesi, Delos Digital, 2016)

Una cerimonia durata meno di un'ora: il corpo lavato, avvolto in un lenzuolo bianco, infilato in un sacco e fatto scivolare nel Golfo Persico da una tavola inclinata. Come si usava per i pirati e per tutti i morti in mare. Ma il funerale da marinaio per questo guerrigliero che tutta la vita si è nascosto tra grotte e montagne perplime: e mica poco. Dal mufti d'Egitto al santuario di Al Azhar gli esperti islamici insorgono: la sepoltura al largo è "peccato». (Angelo Aquaro, Ė giallo sui funerali. Gettato in mare per evitare che fosse venerato, "la Repubblica", 3/5/201I)

Come vediamo dagli esempi - una poesia, un articolo di cinema, un romanzo ebook di fantascienza e un articolo di cronaca estera - l'uso sembra non essere più esclusivamente scherzoso, per quanto ancora la maggior parte dei contesti in cui si ritrova il verbo siano di registro colloquiale e la percezione dei parlanti e degli scriventi sia ancora legata agli sketch comici di Guzzanti:

\footnotetext{
Notare tanta passione per la questione mantello [...] dovrebbe perplimere - per dirla alla Guzzanti - e non invece appassionare una comunità che ha occhi per guardare e capacità per discernere quel che è importante da quello che realmente non solo non lo è ma, al contrario, non andrebbe neanche considerato oggetto su cui valga la pena soffermarsi. (Eleonora Urzi Mondo, Messina. Accorinti: l'antieroe col mantello che indigna, www.lecodelsud.it, Io/I/2017)
}

A conferma della lentezza con cui sia va acclimatando il verbo nel lessico italiano vi sono le oscillazioni rilevate delle forme flesse al passato remoto, perplessi, perplimei e perplimetti, e, caso ancor più curioso, al participio passato. Per quanto riguarda il passato remoto le attestazioni in rete confermerebbero una prevalenza per la forma perplessi (su Google il 2I/2/202I: 67 risultati per mi perplessi, 37 per perplimei e 24 per perplimetti), analogamente a quanto accade per il verbo opprimere, che presenta la forma oppressi, più comune, a fianco alle varianti opprimei e opprimetti, più rare (cfr. DOP). Stupisce invece la presenza, sebbene sporadica, delle forme al participio passato perplimito (8 risultati su Google) e perplimuto (I97 risultati), naturalmente accanto al comune perplesso da cui il verbo deriva; 
ma come fa notare il Garzanti 2017 "bisogna tener presente che l'uso di questo verbo è scherzoso e chiunque ci si può sbizzarrire" e forse le forme intendono differenziare l'uso del participio come verbo da quello come aggettivo.

\section{Cita come:}

Luisa di Valvasone, Significato e origine di perplimere: nota di aggiornamento , "Italiano digitale", 2021, XVI, 2021/1 (gennaio-marzo)

DOI: $10.35948 / 2532-9006 / 2021.5483$

Copyright 2021 Accademia della Crusca

Pubblicato con licenza creative commons CC BY-NC-ND 